\title{
Uncovering bidirectional ecological associations from co-occurrence and environmental data
}

\author{
Sara Si-moussi ${ }^{1}$, Esther Galbrun ${ }^{2}$, Mickael HEDDE $^{3}$, and Wilfried Thuiller ${ }^{4}$ \\ ${ }^{1}$ INRA Centre de Montpellier \\ ${ }^{2}$ University of Eastern Finland School of Computing Kuopio \\ ${ }^{3}$ INRA Montpellier Research Centre \\ ${ }^{4}$ Laboratoire d'Ecologie Alpine
}

May 5, 2020

\begin{abstract}
The interplay between environmental suitability, dispersal and biotic interactions induces spatial patterns of species' coabundance. Existing statistical frameworks that infer the underlying interactions from these patterns either ignore the species response to the environment or they fail to account for the asymmetric nature of interactions. Here, we propose a framework that (a) models pair-wise associations as directed influences from a source to a target species, parameterized with two species-specific latent variables: the response of the target species to the community, and the effect of the source species on the community; and (b) jointly fits these associations with a habitat suitability model through a conditional abundance model. Using both simulated and empirical data, we demonstrate the ability of the framework to recover known associations and highlight the properties of the learned association networks. Our framework should now pave the way for getting more accurate pictures of interspecific dependencies from empirical data.
\end{abstract}

\section{Hosted file}

main.pdf available at https://authorea.com/users/301502/articles/431359-uncoveringbidirectional-ecological-associations-from-co-occurrence-and-environmental-data 
Learning bidirectional ecological associations from co-occurrence and environmental data

\title{
Supplementary Materials
}

\author{
Sara Si-moussi, Esther Galbrun, Mickael Hedde, Wilfried Thuiller
}

February 27, 2020

\begin{abstract}
Contents
1 Supplements to framework description

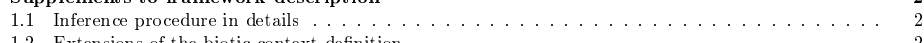

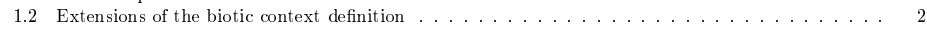

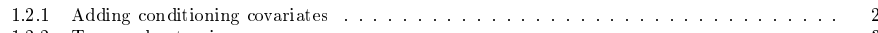

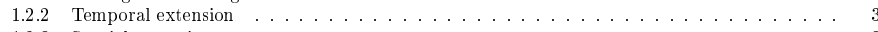

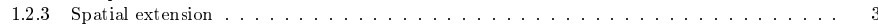

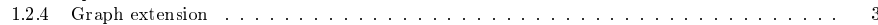

2 Supplements to the simulation experiment

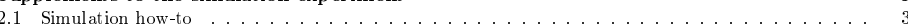

2.1 .1 Notation . . . . . . . . . . . . . . . . . . 4

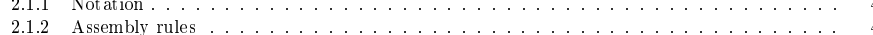

2.2 Simulation

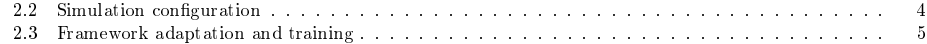

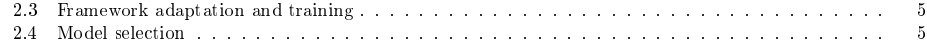

2.4 Model seledicin

Supplements to the empirical application

$\begin{array}{ll}3.1 & \text { Environmental data preparation } \\ 3.2 & \text { Framework adaptation and training }\end{array}$

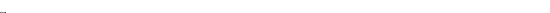

3.3 Embedding dimension and lasso parameter selection $\ldots \ldots \ldots \ldots \ldots \ldots \ldots \ldots$

$\begin{array}{ll}3.4 & \text { Habitat suitability } \ldots \ldots \ldots \ldots \ldots \ldots \ldots \ldots \ldots \\ 3.5 & \text { Analyzing the functional meaning of plant embeddings } \ldots \ldots \ldots \ldots \ldots \ldots \ldots\end{array}$

4 Discussion of the offset choice

4.1 Interplay of offset choice, niche overlap and carrying capacity $\ldots \ldots \ldots \ldots \ldots \ldots \ldots$

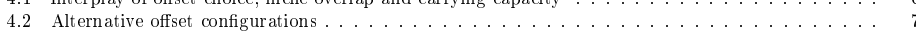

